\title{
O ping-pong da pessoa mais velha: (Re)pensar a integração de Cuidados
}

\section{Ping-pong of the older adult: (Re)think integrated care}

\author{
Maria Inês Espírito Santo \\ Adelaide Belo $(3,4)$ \\ Vera Almeida $(3,5,6)$ \\ Carlos José Russo $(3,7)$

\section{Cátia Gaspar (8)} \\ (1) Instituto Universitário de Lisboa (ISCTE), Portugal \\ (2) Centro Hospitalar Universitário de Lisboa Central (CHULC), Portugal \\ (3) Portuguese Association for Integrated Care (PAFIC), Portugal \\ (4) Unidade Local de Saúde Litoral Alentejano, Portugal \\ (5) Associação Portuguesa de Administradores Hospitalares, Portugal \\ (6) Hospital Garcia de Orta, Portugal \\ (7) Unidade de Saúde Familiar Conde de Oeiras, Portugal \\ (8) Centro Hospitalar Universitário de Lisboa Norte (CHULN), Portugal
}

Recebido: 19/10/2021; Revisto: 25/10/2021; Aceite: 07/11/2021.

*Estudo apresentado no Portuguese Association of Integrated Care (PAFIC), VIII Conferência de Valor da Associação Portuguesa de Administradores Hospitalares, 2020

\section{Resumo}

Contexto e Objetivo: Este estudo visou perceber e analisar as experiências e o conhecimento de vários profissionais de saúde, da área social e de um cuidador informal sobre a integração de cuidados à pessoa mais velha num contexto pandémico. Método: realizou-se uma investigação de caráter qualitativo na qual participaram sete participantes com funções distintas, mas complementares na essência do cuidado. A recolha de dados foi feita através das comunicações dos participantes do seminário "O ping-pong dos nossos idosos - (Re)pensar, (re)construir e Integrar Cuidados" promovido pela Portuguese Association of Integrated Care (PAFIC) no âmbito da VIII Conferência de Valor da Associação Portuguesa de Administradores Hospitalares (APAH). Resultados: Na perspetiva dos participantes, a integração de cuidados no percurso da pessoa é fundamental e para isso é necessário estreitar a articulação entre Serviço Nacional de Saúde e o social. Bem como, centrar os cuidados na pessoa, aliada à matriz comunitária que leve à transformação do modelo organizativo vigente de cuidados e à mudança nos modelos de gestão que se mantém inalterados há muitos anos. A integração de cuidados das pessoas não depende só da atividade da área da saúde, mas também é condicionada por outros setores de atividade, e por isso importa avaliar o papel que a sociedade civil pode assumir na prestação de cuidados. Conclusões: A pandemia COVID-19 trouxe a oportunidade de reflexão sobre o cuidado à pessoa mais velha e como se podem potenciar parcerias e estratégias de articulação entre os diferentes níveis de cuidados, saúde, sociais e comunitários.

Palavras-Chave: Integração de Cuidados; Pessoa mais velha; Multidisciplinariedade; Desafios sociais.

\section{DI\&D | ISMT}

rpics@ismt.pt

https://rpics.ismt.pt

\section{Publicação em Acesso Aberto}

(c) 2021. O(s) Autor(es). Este é um artigo de acesso aberto distribuído sob a Licença Creative Commons Attribution, que permite uso, distribuição e reprodução sem restrições em qualquer meio, desde que o trabalho original seja devidamente citado.
Maria Inês Espírito Santo 


\begin{abstract}
Background and Aim: This study aimed to understand and analyze the experiences and knowledge of various health professionals, the social area and an informal caregiver on the integration of care for the elderly in a pandemic context. Method: a qualitative investigation was carried out in which seven participants with distinct but complementary roles in the essence of care participated. Data collection was carried out through the communications of the participants of the seminar "The ping-pong of our elderly - (Re)think, (re)build and Integrate Care" promoted by the Portuguese Association of Integrated Care (PAFIC) within the scope of the VIII Value Conference of the Portuguese Association of Hospital Administrators (APAH). Results: From the participants' perspective, the integration of care in the person's path is fundamental, and for this, it is necessary to strengthen the articulation between the National Health Service and the social. As well as centering care on the person, allied to the community matrix that leads to the transformation of the current organizational model of care, to the change in management models that have remained unchanged for many years. The integration of people's care depends not only on the activity in the health area but is also conditioned by other sectors of activity, and therefore it is important to assess the role that civil society can play in providing care. Conclusions: The COVID-19 pandemic allowed the reflection on the care of the older people and how partnerships and articulation strategies between the different levels of care, health, social and community could be enhanced.
\end{abstract}

Keywords: Integration of Care; Older adult; Multidisciplinarity; Social challenges.

\title{
Introdução
}

O seminário "O ping-pong dos nossos idosos: (re)Pensar, (re)Construir e Integrar Cuidados" inseriu-se na oitava Conferência de valor da Associação Portuguesa de Administradores Hospitalares. Com este evento, pretendeu refletir-se e identificar-se um diagnóstico sobre a integração de cuidados entre a saúde, o setor social e a comunidade na resposta às necessidades da pessoa mais velha. E simultaneamente, contribuir para apontar caminhos inovadores que coloquem o processo assistencial centrado na pessoa e nas suas famílias, respeitando o seu percurso de vida.

Portugal é um dos países europeus em que nos próximos anos mais se fará sentir na pressão do envelhecimento populacional, no aumento da procura de unidades de saúde, com particular relevo nas doenças crónicas não transmissíveis (Coelho et al., 2019). Assim, o envelhecimento demográfico, a cronicidade da doença e concomitantemente a fragmentação dos cuidados entre os diferentes níveis de serviços, constituem desafios ao Serviço Nacional de Saúde (SNS) português. A articulação efetiva entre os diferentes níveis de cuidados de saúde e sociais é imperiosa na elaboração de um plano participado e integrado que permita que o cuidado se efetue de forma estruturada e normalizada, assegurando a sua continuidade com qualidade e segurança.

O conjunto de fatores que tem vindo a influenciar o paradigma nos serviços de saúde e a consequente necessidade de mudança de estratégia global tem vindo a subjazer um modelo integrado de cuidados entre os diversos níveis de saúde e social centrado na pessoa (Organização Mundial de Saúde [OMS], 2015). A integração de cuidados de saúde deverá ser uma prioridade de forma a garantir cuidados sustentáveis e de elevada qualidade (Goodwin et al., 2012). Este tema tem sido amplamente discutido e debatido a nível político por vários governos, a nível nacional e internacional para a necessidade de práticas de gestão conscientes e efetivas, com uma forte aposta na construção de relações 
interprofissionais e na partilha de informações clínicas, reconhecendo que a mudança ao nível da integração de cuidados é um processo de aprendizagem e evolução (Tsasis et al., 2012).

Neste sentido é necessário que o caminho a percorrer tenha como linha orientadora quatro grandes objetivos (Marques, 2012): 1) Sustentabilidade do sistema: porque só um sistema sustentável será capaz de responder às necessidades reais da população; 2) Acessibilidade dos doentes/utentes ao promover o acesso aos cuidados de saúde adaptados às necessidades reais das pessoas; 3) Qualidade: como padrão de exigência em qualquer nível de saúde; 4) Relação profissional-doente: como reforço e articulação entre os níveis de cuidados de saúde e sociais garantindo as melhores respostas e encaminhamento adequado.

Considerando esta organização de cuidados de saúde é fundamental que a articulação entre cada um dos prestadores seja eficaz na partilha de informação cujo objetivo vise a implementação, monitorização e avaliação entre os diferentes níveis de cuidados de saúde e setor social numa perspetiva biopsicossocial. São aspetos fulcrais para uma melhor racionalização e potencialização dos recursos humanos e financeiros, conduzindo a uma coordenação de cuidados eficazes e integrados que levam a uma redução de custos significativos (Torres, 2011).

De acordo com as orientações do Plano Nacional de Saúde entre 2011-2016 da Direção Geral de Saúde (DGS, 2012), a implementação de um programa integrado de alta hospitalar tem:

"ganhos adicionais em saúde através da redução das desigualdades em saúde, de forma transversal ou focada a grupos vulneráveis (...), melhora a sua efetividade e se disseminam como boas práticas (...). A articulação em cada nível de cuidados, entre níveis e entre sectores, no sentido de assegurar a gestão integrada dos recursos intra-hospitalares e gestão de altas são processos promotores de equidade e acessibilidade que incluem: a transferência de cuidados gerais para os Cuidados Primários e para a comunidade (...) ou o planeamento da alta hospitalar o mais precocemente possível" (p. 6).

Estas orientações pretendem igualmente:

"desenvolver e protocolar a articulação de cuidados e investir de forma proactiva na comunicação entre prestadores dentro e entre instituições e serviços, assegurando a articulação de cuidados e orientando o doente de forma eficiente, e permitir a monitorização e avaliação" (DGS, 2012, p. 15).

Assim, foi objetivo do nosso estudo perceber e analisar as experiências e o conhecimento de vários profissionais de saúde, da área social e de um cuidador informal sobre a integração de cuidados à pessoa mais velha num contexto pandémico.

\section{Método}

\section{Participantes}

O estudo foi constituído por sete participantes com funções distintas, mas complementares na essência do cuidado. Tal como indicado no Quadro 1, a heterogeneidade e complementaridade do perfil do cuidador formal e informal traz ao estudo algo invulgar, mas crucial e foi com base nesses critérios que recaiu a escolha dos participantes: a dimensão do conhecimento, a diferenciação técnica e a experiência 
dos elementos envolvidos na prestação de cuidados, que vai desde o familiar aos diferentes profissionais do terreno, seja da área da saúde, seja do social. Um olhar que se perspetiva como integrado.

\section{Quadro 1}

Caracterização dos Participantes Quanto ao seu Papel e Relevância na Integração no Estudo

\begin{tabular}{ll}
\hline Cuidador (papel) & Relevância na integração \\
\hline Informal & Filha de utente institucionalizada, Profissional de saúde \\
Formal & Diretor de um serviço de medicina em hospital, Médico \\
Formal & Médico de Medicina Geral de Família e Diretor clínico de uma IPSS \\
Formal & Assistente Social em contexto hospitalar \\
Formal & Diretora Técnica e Assistente Social de uma ERPI \\
Formal & Enfermeira numa ERPI \\
Formal & Ajudante familiar numa ERPI \\
\hline
\end{tabular}

Nota. IPSS = Instituição Particular de Solidariedade Social; ERPI = Estrutura Residencial para Idosos.

\section{Tipo de Estudo e Procedimento}

O estudo foi exploratório e descritivo, estruturado em relatos de experiência e de análise reflexiva de seis profissionais das áreas da saúde e social e de um cuidador familiar, que participaram no seminário de debate.

A análise das experiências e vivências formais e informais do cuidado exige abordagens qualitativas que compreendam a precisão e o olhar sistémico da integração do cuidado. Para perceber a diversidade de singularidades e permitir o conhecimento sobre os temas, as vivências que os profissionais experienciaram foram relatadas com caráter interdisciplinar, na complementaridade com o testemunho de um cuidador informal de uma pessoa institucionalizada em Estrutura Residencial para Idosos (ERPI).

Os participantes foram informados dos objetivos e das condições de participação no presente trabalho, tendo declarado sentirem-se esclarecidos e aceitado participar de forma voluntária, autorizando e disponibilizando o conteúdo narrativo para fins de publicação.

A codificação dos participantes, para evitar a sua identificação, foi feita de E1 a E7.

A análise de conteúdo categorial das respostas a questões abertas seguiu a metodologia de Bardin (2006), na constituição de três fases: pré-análise, exploração dos dados e tratamento dos resultados - inferência e interpretação. As narrativas abordaram as seguintes dimensões: 1) diagnóstico do processo assistencial na resposta às necessidades das pessoas mais velhas; 2) exemplos de boas práticas/experiências do terreno; e 3) perspetivas para o futuro. 


\section{Resultados}

Dos resultados obtidos das comunicações emergiram consensos que nos permitiram considerar três fatores importantes: 1) os lares devem ser a última etapa no processo de apoio aos mais velhos; 2) a multidisciplinariedade é fulcral, bem como preconizar o trabalho no terreno e o envolvimento das pessoas; 3) a integração de cuidados deveria ser uma prioridade política pelos ganhos obtidos, considerando-se para o efeito a importância da articulação entre os diferentes níveis de cuidados: saúde, sociais e comunitários.

\section{Diagnóstico do processo assistencial}

O envelhecimento demográfico aliado ao aumento progressivo da esperança média de vida e do aumento da dependência funcional, constituem necessidades adicionais dos cuidados de saúde em Portugal. Para além disso, a grande maioria das altas hospitalares são de pessoas com mais de 65 anos que não necessitam de uma intervenção dirigida à cura de uma situação aguda. As pessoas mais velhas necessitam, sim, de uma nova abordagem integrada que inteire o sistema de saúde e das organizações sociais, direcionadas para o indivíduo com necessidades de apoio nas atividades básicas de vida e nos cuidados de reabilitação e de reinserção (Costa, 2009; Escoval et al., 2010).

Verificámos nos discursos dos participantes que a longevidade da população reflete conquistas e desafios. No entanto, a COVID-19 veio evidenciar o agravamento de potenciais consequências negativas:

"O envelhecimento não é, nem pode ser, um problema. Viver mais é maravilhoso. Mas para isso, precisamos de políticas sustentáveis e realistas. Precisamos de mudar o pensamento, sensibilizar, informar que uma pessoa com mais de 65 anos de idade é um cidadão de direitos, que pode e deve continuar a contribuir e a participar ativamente na sociedade. Cada pessoa idosa tem uma bagagem de experiências, conhecimentos, vivências que em muito nos podem enriquecer..." (E5)

"A pandemia forçou o emergir de outras vulnerabilidades, vulnerabilidades já existentes das pessoas idosas. A solidão, o abandono, a negligência, as desigualdades no acesso aos cuidados de saúde, pensões baixas, habitações precárias, deficits de/das respostas sociais veio expor fragilidades do cuidar, um cuidado desfragmentado..." (E2; E5)

"Uma das fragilidades do Sistema de Saúde que esta pandemia destacou foi a sua fragmentação, a falta de comunicação entre os vários níveis de cuidados, a falta de coordenação e a excessiva centralização. Esta realidade foi das maiores dificuldades que enfrentamos nesta crise, a qual gerou disfunções na resposta e nos obrigou a ter de improvisar. Esta deveria ser uma das maiores lições que devíamos retirar da gestão desta pandemia." (E1)

"Os nossos hospitais não estão preparados para o internamento de pessoas idosas, quer pela sua arquitetura, quer a sua prática assistencial em que não é acautelada a perda da sua funcionalidade e da sua autonomia quanto à valorização e preservação da integridade cognitiva e emocional. Uma pessoa idosa, previamente autónoma e com capacidade funcional na realização das suas AVD's, internado durante dois a três dias para comprometer a sua funcionalidade e autonomia. Na generalidade, não existe uma preocupação em olhar para a pessoa no seu todo, um olhar integrado de vários saberes." (E5) 
Impera por isso a

"(...) necessidade de um modelo de apoio aos idosos mais equitativo no acesso em igualdade de circunstâncias a todos os serviços, pois é sabido que os idosos com menos recursos económicos estão mais expostos a situações de fragilidade e precaridade de apoio a vários níveis, nomeadamente no acesso à saúde e equipamentos sociais, pois a limitada contratualização com a segurança social coloca as instituições numa situação de fragilidade e sustentabilidade económica/financeira, e por sua vez social, sem capacidade para poder reconhecer salarialmente os profissionais que trabalham neste setor e tornar o mesmo mais atrativo do ponto de vista profissional. Apresentando assim dificuldade em contratar e manter profissionais com competência profissional dado ser pouco atrativo trabalhar no terceiro setor, é necessária uma aposta no quadro de pessoal técnico das ERPI'S, pois estas cada vez acolhem mais idosos com grande dependência e necessidade de serviços profissionalizados." (E2)

Estas limitações e necessidades são sentidas por outro participante.

"(...) Quando somos médicos assistentes de uma ERPI, apercebemo-nos que, mesmo sendo considerado uma estrutura residencial dos nossos idosos, entendemos isto como domicilio (sic) e não existe apoio por parte das unidades de saúde locais na gestão dos cuidados destes residentes, tornando tudo mais complexo e deficitário nas ERPI. Não esta terem médicos, pois na verdade não são "Residências Assistidas", nem unidades de "Cuidados Continuados", o que torna ainda mais precária as situações destes residentes aquando perdem a sua autonomia funcional para se deslocar às unidades locais de cuidados." (E3)

O conjunto de limitações referidos converge para algumas questões/ observações importantes a refletir:

"Não há um cumprimento das leis que fazem referência aos cuidados dos idosos: Lei do estatuto do maior acompanhado - Lei 49/2018; de como são geridas as suas pensões; quem assina os contratos e os consentimentos para as intervenções em saúde e sobre a sua vida e património. Existe, no geral, uma falta de consciência moral na nossa sociedade sobre os cuidados a ter com estes residentes, assim como das suas necessidades. Falta de uma cultura universitária na articulação com estas instituições, a sociedade exige uma total responsabilidade ao estado na gestão destes cuidados." (E3)

"Deste conjunto de limitações e necessidades, a disparidade financeira representa uma preocupação. Ou seja, existem por exemplo (...) assimetrias financeiras entre as instituições do litoral e do interior, assimetrias estas que podem influenciar a qualidade dos serviços prestados, nomeadamente na contratualização de recursos humanos que permitam ter uma equipa multidisciplinar, devendo haver diferenciação positiva entre as mesmas de forma equitativa diminuindo estas discrepâncias." (E2)

Está ainda patente no discurso da filha da utente institucionalizada a necessidade preventiva e antecipatória das respostas:

"Passei por várias fases na integração institucional do meu familiar. Adaptei-me a um padrão de visita e integração familiar depois da institucionalização e adicionalmente a gerir a relação emocional de maior afastamento familiar ao longo do tempo. Mas a grande tranquilidade surge quando é manifesta uma oferta permanente de apoio na saúde e no apoio social de forma interdisciplinar, controlada e diferenciada..." (E4)

Partindo deste testemunho: 
"(...) urge alterar o paradigma da visão paternalista para uma lógica mais centrada na pessoa como premissa para a salvaguarda da autonomia, liberdade, escolha, autodeterminação e dignidade." (E5) "A necessidade de integração de cuidados é um desafio cada vez mais premente. Não é possível continuar a tratar os doentes crónicos complexos desta forma fragmentada, episódica, reativa, através das urgências e focados na doença. Esse é um caminho apontado por múltiplos organismos internacionais, entre eles a Organização Mundial de Saúde (OMS), caminho que é já uma prioridade em muitos países." (E1)

\section{Boas Práticas ou Experiências no Terreno}

Face ao diagnóstico do processo assistencial foram identificados pelos participantes algumas estratégias para melhorar a integração de cuidados, bem como o reconhecimento de boas práticas a replicar:

"(...) a necessidade de uma nova arquitetura dos cuidados com a implementação de mais sistemas locais de saúde com gestão conjunta, liderança de orçamento único dos vários níveis de cuidados e da assistência social. Ainda a introdução da integração de cuidados na contratualização com hospitais e cuidados primários; concentrar o internamento e descentralizar o ambulatório na rede hospitalar; repensar a reforma dos cuidados primários; criar incentivos para resultados conjuntos entre hospitais e centros de saúde; contratar profissionais para os programas de integração de cuidados, em particular, mais internistas, que são os interlocutores naturais para os médicos de família dentro dos hospitais; investir nos sistemas de informação e comunicação, no processo clínico único e na telemedicina; melhorar a literacia, promover campanhas de educação para ajudar os cidadãos a gerirem melhor a sua saúde, mas também a utilizar melhor os serviços de saúde."(E1)

Nesta lógica, verifica-se a importância do:

"(...) planeamento de alta hospitalar como um dos principais instrumentos de gestão, eficácia e qualidade da prestação de cuidados, que se pretende que seja o mais precoce possível, coordenado e integrado com todos os elementos da equipa de saúde em que a pessoa e a família participam ativamente. $\mathrm{O}$ assistente social tem um papel preponderante no sistema de saúde com uma abordagem proativa e não reativa na identificação das necessidades das pessoas e definir o mais precoce possível o plano de alta, seja através de acolhimento ou triagem sistemática por indicadores de risco, por forma a minimizar o tempo de protelamento da alta hospitalar, através da gestão equilibrada da complexidade dos problemas sociais. Mas o papel do Serviço Social não fica só pelas portas de um hospital. As suas competências abrangentes, comunitárias e inclusivas são essenciais para abordar as determinantes sociais da saúde e minimizar ou eliminar as desigualdades estruturais preexistentes à pandemia." (E5)

Paralelamente,

"(...) temos um longo caminho a percorrer ao nível da inovação, dinamização do serviço de apoio domiciliário e, acima de tudo, o reconhecimento dessa necessidade por parte dos serviços da Segurança Social que ainda continua muito focada/ centrada nos serviços tradicionais (alimentação, tratamento de roupa...). Assistimos a uma procura crescente de serviços diferenciados, porque verificamos uma alteração dos hábitos e modus de vida da atual população em envelhecimento e é necessário acompanhar e responder de modo personalizado e individualizado às necessidades emergentes, 
nomeadamente de serviços de profissionais de saúde, tal como por exemplo fisioterapia, enfermagem ao domicílio, acompanhamento a exames e consultas." (E2)

Por isso a importância de:

"(...) antecipar o futuro e apostar na permanência de profissionais de saúde com contratualização em horário completo (ex. enfermeiro em todos os turnos). Com ganhos em saúde: Evitaram idas ao Hospital em todas as situações passíveis de resolução local; diminuíram gastos em saúde; evitaram infeções hospitalares; ocupação de camas; adaptação/Colaboração/Poli- incapacidades conhecidas e desadaptação dos utentes da ERPI; confronto com a Ineficiência (formação): um Assistente Operacional num Hospital é diferente de um Assistente Ação Direta numa ERPI." (E4)

\section{Perspetivas para o Futuro}

"Para prosseguir neste caminho é preciso estar aberto a aprender realmente com o que está a acontecer, ter visão estratégica e coragem para reformar. É preciso que a integração de cuidados se torne uma prioridade política, tal como aconteceu com a implementação da hospitalização domiciliária, que foi das reformas mais rápidas e extensivas que conheço a nível internacional. Se há um mito que caiu com esta pandemia é que não somos capazes de mudar." (E1)

"Um outro caminho passa por uma maior articulação entre serviços e entidades, nomeadamente, saúde, segurança social, IPSS (Instituição Particular de Solidariedade Social) e comunidade. A ausência de um planeamento estratégico e concertado conduz a uma fragmentação da intervenção, esquecendo que o idoso é um ser biopsicossocial. É necessária uma intervenção articulada por forma a evitar o "PING PONG" dos mais velhos de serviço em serviço ..." (E2)

Para que possam ultrapassar e gerir as dificuldades do processo de mudança, é necessário:

"(...) que os bons resultados devam ser valorizados, por incentivos atrativos que não só os salariais; por exemplo, a formação contínua, uma profissão com mecanismos atrativos (horários, benefícios de parcerias com a comunidade por cooperação, criando uma condição prioritária ou facilitadora, sejam elas nas autarquias, na comunidade, nos centros de saúde, no comportamento social, seguros, pequeno comércio, agências de viagens, museus, teatros, cinemas, áreas comerciais, para estes profissionais." (E4)

"(...) algumas soluções de desenvolvimento de políticas sociais sobre estas instituições e as necessidades das pessoas idosas como autonomia moral e funcional diminuídas seriam bastante interessantes assim como fazer uso das leis existentes. Sugestões:

1) Circuitos de articulação com os centros de saúde, inscrever a todos os residentes no centro de saúde da área onde está inserida a ERPI (Estrutura Residencial para Idosos), criando protocolos e estatutos para a articulação com os hospitais de referência da unidade local de cuidados;

2) Melhoria dos contratos e da valorização do emprego nas ERPI: médico, enfermeiros, auxiliares.

3) Capacitação social e consciência moral sobre a responsabilidade como comunidade (é uma responsabilidade de todos) - comunidades compassivas, cuidar em rede;

4) Ser conscientes da vulnerabilidade;

5) Incluir as ERPI nos estágios profissionais e nas universidades; 
É preciso um cuidado integrativo, em rede, que possa incluir a todos, desde escolas, empresas e outras estruturas presentes na área da ERPI. Desenvolver um modelo que acorde a comunidade:

1) Modelo da atenção centrada na pessoa;

2) Envolver as comunidades nos cuidados das doenças que causam grande dependência, como são as demências, outras doenças que provocam alterações e deterioração do estado cognitivo e as do foro da saúde mental." (E3)

Há necessidade de normalizar a sociedade de forma séria junto dos idosos e dos profissionais. A comunidade em geral deve envolver-se em parcerias com contributos válidos junto dos idosos em estruturas residenciais. Por exemplo:

"Trazer a comunidade às ERPI. Criar o estatuto de linha da frente para o idoso, fazer parte dele e isso ser mérito com reconhecimento. Em contrapartida, a comunidade terá benefícios governamentais (por exemplo fiscais);

1) Fluxos facilitadores interministeriais - resolve burocracia, torna céleres processos comuns;

2) Vinda de alunos tutoriais - resolve o mecanismo de colaboração supervisionada, liberta profissionais para pausa ou ausência;

3) Outros fluxos organizacionais com a comunidade: resolve a alteração comportamental face ao envelhecimento crescente, aumenta a divulgação empresarial comunitária e o seu contributo social". (E4)

Para que o cuidado integrado seja implementado, é fundamental estreitar a articulação entre saúde e social e centrar de forma efetiva os cuidados na pessoa, aliada à matriz comunitária que leve à transformação do modelo organizativo vigente de cuidados e à mudança nos modelos de gestão que se mantêm inalterados há muitos anos.

"Atravessamos uma janela de oportunidade ímpar para que trabalhemos em conjunto com o mesmo propósito, a pessoa. E é nesta perspetiva construtiva e de mudança de paradigma que temos de dar o salto, unindo esforços, propostas e estratégias de caráter preventivo no diagnóstico das situações das pessoas mais velhas e no acompanhamento no seu percurso de vida. Por isso, defendo a existência de assistente social de família integrado nas equipas de saúde, no acompanhamento dos agregados familiares nos seus diferentes ciclos de vida, de trabalhar nas determinantes sociais, na literacia em saúde, na realização de um diagnóstico e tratamento precoce da doença e das necessidades e de uma gestão adequada da doença crónica. Estes profissionais, pelas suas competências, devem fazer parte desta equação por um cuidado pautado por uma gestão eficaz de cuidados humanizados, integrados e de bem-estar para a pessoa idosa." (E5)

Neste processo que se perspetiva de mudança, a certificação pode ser considerada como um instrumento de extrema importância na melhoria da qualidade dos cuidados.

“(...) A certificação da qualidade das respostas sociais de apoio à terceira idade devia ser instituída (sic) como obrigatório, mas gratuito, pois esta certificação permitiria às instituições planear, executar e avaliar os serviços prestados, monitorizando continuamente a qualidade dos mesmos e permitindo ainda aos idosos e seus familiares terem padrões de referência de qualidade para se candidatarem a respostas sociais." (E2) 


\section{Discussão}

O conjunto de propostas estratégicas dos participantes converge com o argumento de (Gröne \& GarciaBarbero, 2001) que sustentam que a integração de cuidados agrega vários conceitos, tais como a distribuição, gestão e organização de cuidados relacionados com o diagnóstico, tratamento, cuidados, reabilitação e promoção da saúde.

A integração dos cuidados primários, secundários e de terceiro nível é fundamental, bem como de outros sectores, e, particularmente, do sector social. A saúde das populações não depende só das atividades do sector da saúde, mas está também bastante condicionada por outros sectores de atividade. Importará também avaliar o papel que a sociedade civil pode assumir no processo de prestação de cuidados. E será importante também discutir, para além da integração vertical - interinstitucional, as possibilidades de integração horizontal - intrainstitucional (e.g., no hospital, entre várias especialidades). Esta mudança de paradigma pressupõe um novo comportamento ou atividade, uma mudança estratégica da organização, que conduza a desempenhos mais elevados, alimentada pela criatividade das pessoas e apoiada por elas, com uma cultura que valorize a melhoria contínua" (Carapeto \& Fonseca, 2006, pp. 348-349). Aliada a esta condição de mudança é importante saber como se integram cuidados e como se combinam estratégias e recursos. Teremos de encontrar e pensar formas de integrar a prevenção, o diagnóstico, o tratamento e a reabilitação, rever os padrões e os critérios de referenciação de doentes, integrar a informação dos utentes, definir quem envolver no plano de cuidados e até rever os perfis de competências dos profissionais, eventualmente criando espaço para o surgimento de novas profissões, bem como para a revisão dos atuais perfis de competências dos diferentes profissionais (Shaw et al., 2011). Com efeito, alcançar cuidados integrados requer envolver no planeamento e prestação de cuidados "a perspetiva do utente como organizador principal da prestação de serviço" (Lloyd \& Wait, 2006, p. 7).

Para que as estratégias de cuidados integrados sejam bem-sucedidas, é fulcral envolver todos os stakeholders (utentes, prestadores de cuidados, responsáveis pelo planeamento, instituições), mas as estratégias conducentes a melhorar os cuidados ou os serviços prestados aos utentes poderão levar a perdas ao longo do sistema, nomeadamente de poder e controlo de alguns profissionais e/ou instituições, o que gerará possíveis resistências (Gröne \& Garcia, 2002).

\section{Conclusão}

Este artigo procura alertar para a necessidade de mudança de um paradigma de cuidados estagnado para um que preconize uma abordagem biopsicossocial na melhoria da articulação entre os diferentes níveis de cuidados. Para que se concretize uma melhoria da eficiência e satisfação dos utentes e da qualidade dos cuidados e, consequentemente, no desenvolvimento das competências profissionais. E por isso, a relevância do presente artigo, uma vez que traz a visão e contributos dos vários stakeholders na prestação dos cuidados de saúde, social e informal.

Os tempos de COVID-19 devem fazer refletir sobre as oportunidades e desafios das novas parcerias de trabalho estabelecidas e sobre como podemos integrá-las na prática profissional, potenciando a diversificação de serviços ao cidadão e a sua continuidade. E assim se pode construir um caminho de 
integração de cuidados baseado em Valor para a pessoa mais velha e, por consequência, com mais ganhos em Saúde.

Agradecimentos | Acknowledgements: Os autores agradecem a todos os palestrantes que participaram no seminário "O ping-pong dos nossos idosos - (Re)pensar, (re)construir e Integrar Cuidados" promovido pela PAFIC - Portuguese Association of Integrated Care no âmbito da oitava Conferência de Valor da Associação Portuguesa de Administradores Hospitalares (APAH) pelos contributos e conhecimento.

Conflito de interesses | Conflict of interest: nenhum | none.

Fontes de financiamento | Funding sources: nenhuma | none.

Contributos: MIES: Revisão da literatura; Recolha, inserção e tratamento dos dados; redação do manuscrito. AB, VA, CJR, CG: Contributo na redação do manuscrito; Tratamento e discussão dos dados; Revisão da redação final do manuscrito

\section{Referências}

Bardin, L. (2006). Análise de conteúdo. Edições 70.

Carapeto C., \& Fonseca F. (2006). Administração pública - modernização, qualidade e inovação (2a ed.). Lisboa.

Coelho, A., Catalão, P., \& Nunes, N. (2019). Doenças não transmissíveis em Portugal: Desafios e oportunidades. Anais do Instituto de Higiene e Medicina Tropical (Supl. 1), 17-21. https://doi.org/10.25761/anaisihmt.317

Costa, M. (2009). Interface do hospital com os cuidados continuados integrados. Em L. Campos, M. Borges, \& R. Portugal (eds.), Governação dos hospitais (pp. 349-364). Casa das Letras.

Direção Geral de Saúde. (2012). Plano Nacional de Saúde 2012-2016 - Enquadramento do Plano Nacional De Saúde. https://bit.ly/3qwOEZQ

Escoval, A., Fernandes C, A., Matos, T., \& Santos, A. (2010). Plano Nacional De Saúde 2011-2016: Cuidados de saúde hospitalares. Escola Nacional de Saúde Pública. https://bit.ly/3wCLxPp

Goodwin, N., Smith, J. A., Davies, A., Perry, C., Rosen, R., Dixon, A., Dixon, J., \& Ham, C. (2012). Integrated care for patients and populations: Improving outcomes by working together. A report to the Department of Health and NHS Future Forum. The King's Fund. https://bit.ly/3DOZskl

Gröne, O., \& Garcia-Barbero, M. (2001). Integrated care. A position paper of the WHO European office for integrated care services. International Journal of Integrated Care, 1(2), 1-10. http://doi.org/10.5334/ijic.28

Gröne, O., \& Garcia-Barbero, M. (2002). Trends in integrated care - Reflections on conceptual issues (EUR/02/5037864). World Health Organization, Copenhagen.

Lloyd, J., \& Wait, S. (2006). Integrated care: A guide for policymakers. Alliance for Health and the Future. https://bit.ly/30856mP

Marques, A. (2012). A rede nacional de urgência e emergência - reflexões no contexto da reforma hospitalar em 2011. Grupo Técnico para a Reforma Hospitalar.

Organização Mundial de Saúde. (2015). Relatório mundial sobre envelhecimento e saúde. Organização Mundial de Saúde. https://bit.ly/3mOfBEt

Shaw, S. E., Rosen, R., \& Rumbold, B. (2011). What is integrated care? An overview of integrated care in the NHS [Relatório]. Nuffieldtrust. https://bit.ly/3kcOfbm

Torres, A. (2011). A articulação de cuidados de saúde primários e hospitais e seu impacto a nível regional [Apresentação de artigo]. $17^{\circ}$ Congresso APDR, Bragança, Portugal. https://bit.ly/3H2ZhaU

Tsasis, P., Evans, J. M., \& Owen, S. (2012). Reframing the challenges to integrated care: A complex-adaptive systems perspective. International journal of integrated Care, 12, e190. https://doi.org/10.5334/ijic.843 